\title{
La revelación social a partir de la guía GRI de la economía social: una herramienta para mejorar las relaciones socio- cooperativa
}

\section{Francisca Castilla-Polo, Dolores Gallardo-Vázquez y Ma Isabel Sánchez-Hernández}

\section{RESUMEN}

La problemática del socio dentro de la cooperativa y su relación con la Teoría de los Stakeholders centra el interés de este trabajo. Considerando no sólo su participación democrática, sino también su potencial participación dentro de diferentes funciones económicas, planteamos la necesidad de una correcta gestión de las relaciones socio-cooperativa. Concretamente, para contribuir a crear y/o mantener estas relaciones proponemos la revelación social y, específicamente, el empleo de la memoria de sostenibilidad al amparo de la metodología GRI específica para la Economía Social. La facilidad de aplicación y los beneficios asociados a su uso parece que compensan sobradamente los costes de su implementación y justifican nuestra propuesta. Como conclusión, podemos afirmar que el empleo de las memorias de sostenibilidad en las cooperativas es una herramienta poderosa para la mejora de las relaciones con sus socios, si bien, evidenciamos las escasas iniciativas abordadas en este tipo de empresas hacia temas de revelación social.

PALABRAS CLAVE: Sociedades cooperativas, stakeholders, revelación social, GRI, Economía Social. CLAVES ECONLIT: M140, M400, P130.

Cómo citar este artículo: CASTILLA-POLO, F., GALLARDO-VÁZQUEZ, D. \& SÁNCHEZ-HERNÁNDEZ, MI. (2015): "La revelación social a partir de la guía GRI de la economía social: una herramienta para mejorar las relaciones socio-cooperativa", CIRIEC-España, Revista de Economía Pública, Social y Cooperativa, 83, 143-168.

Correspondencia: Francisca Castilla-Polo, Profesora Titular de Universidad, Departamento de Economía Financiera y Contabilidad, Universidad de Jaén, fpolo@ujaen.es; Dolores GallardoVázquez, Profesora Titular de Universidad, Departamento de Economía Financiera y Contabilidad, Universidad de Extremadura, dgallard@unex.es, y $\mathrm{M}^{\mathrm{a}}$ Isabel Sánchez-Hernández, Profesora Contratado Doctor, Departamento de Dirección de Empresas y Sociología, Universidad de Extremadura, isanchez@unex.es. 


\title{
La divulgation sociale d'après les directives GRI en économie sociale : un outil pour améliorer les relations entre membre et coopérative
}

RÉSUMÉ : Cette étude s'intéresse à la question du membre au sein de la coopérative et la met en rapport avec la théorie des parties prenantes (stakeholders). Étant donné que le membre participe démocratiquement, mais qu'il peut également prendre part aux différentes fonctions économiques, nous suggérons qu'une gestion correcte des relations entre membre et coopérative est nécessaire. Concrètement, pour contribuer à la création et/ou au maintien de ces relations, nous proposons de recourir à la divulgation sociale et, en particulier, d'utiliser le rapport de développement durable dans le cadre de la méthodologie GRI spécialement prévue pour l'économie sociale. La facilité d'application de la méthode et les avantages qu'elle présente semblent largement compenser son coût de mise en œuvre et justifient notre proposition. Enfin, nous pouvons affirmer que l'utilisation de rapports de développement durable au sein des coopératives est un outil puissant pour l'amélioration de leurs relations avec leurs membres, même si nous tenons à signaler que peu d'initiatives abordées dans ce type d'entreprises s'orientent vers la divulgation sociale.

MOTS CLÉ : Sociétés coopératives, parties prenantes (stakeholders), divulgation sociale, GRI, économie sociale.

\section{Social disclosure based on the GRI guidelines for the social economy: a tool for improving member-cooperative relations}

\begin{abstract}
The problems of the members within the cooperative society and their relation to Stakeholder Theory are the focus of this paper. Considering not only the members' democratic participation but also their potential role in different economic functions, we address the need for proper management of member-cooperative relations. To help create or maintain these relations, we suggest using social disclosure, particularly sustainability reports prepared according to the specific GRI guidelines for the Social Economy. They are easy to apply and the benefits associated with their use would appear to more than offset the costs of implementation, justifying this proposal. In conclusion, we can state that sustainability reporting is a powerful tool for cooperatives to improve their relations with their members, although we have found few social disclosure initiatives in this type of company.
\end{abstract}

KEY WORDS: Cooperative societies, stakeholders, social disclosure, GRI, Social Economy. 


\section{1.- Introducción}

Nuestro estudio se centra en la Teoría de los Stakeholders tratada, entre otros, por Freeman (1984), Donaldson y Preston (1995), Atkinson et al. (1997), Frooman (1999), Harrison y Freeman (1999), Laplume et al. (2008), Orts y Strudler (2009) y Jensen (2010). Concretamente, nos planteamos indagar la forma de mejorar las relaciones que proclama esta perspectiva con cada uno de los colectivos de interés para la organización a través del empleo de la divulgación de información voluntaria.

Para ello, partimos en primer lugar del análisis del concepto stakeholder o grupo de interés. Para Freeman (1984:25) se trata de "aquellos grupos o individuos que pueden afectar o lo están por el logro de los objetivos de la entidad". Johnson y Scholes (2001:193) los identifican como "aquellos individuos o grupos cuyos objetivos dependen de lo que haga la organización y de los que, a su vez, depende la organización". Para Werther y Chandler (2011:35) son tanto grupos como individuos con un interés en las actividades de una empresa. Nos estamos refiriendo, entre otros, a propietarios -actuales y potenciales-, empleados, proveedores, acreedores, clientes, deudores, Administraciones Públicas y público en general. Todos ellos deben ser considerados por la empresa en el desarrollo de su actividad debiendo recibir la máxima aprobación de los diversos grupos de interés con los que se relaciona, lo cual se traducirá en legitimidad para la empresa si actúa de acuerdo con el sistema de valores de tales grupos (Horrach y Socias, 2011).

En segundo lugar, nos dirigimos a un tipo de sociedad muy peculiar, la sociedad cooperativa, donde el socio, como grupo de interés, presenta una problemática especial a tenor de las diferentes superposiciones entre su papel de propietario y diversas funciones económicas en las que participa, como son la de cliente, proveedor, prestamista o empleado. La utilización de la Teoría de los Stakeholders en el ámbito cooperativo ha sido tratada en algunos trabajos como los realizados por Ruíz-Jiménez y García-Martí (2002), Spear (2004), Mook et al. (2007), Gijselinckx (2009), Puentes et al. (2009), Marín et al. (2010), Pedregosa y García (2011), Retolaza y San José (2011) y Server y Capó (2011).

En tercer lugar, aplicaremos nuestro análisis al ámbito de la Responsabilidad Social Empresarial (RSE) por cuanto las sociedades cooperativas incluyen de forma innata una orientación social como vía para satisfacer a sus principales stakeholders, los socios. La Alianza Cooperativa Internacional (ACI) (1995) las define como "una asociación autónoma de personas que se han unido de forma voluntaria para satisfacer sus necesidades y aspiraciones económicas, sociales y culturales en común, mediante una empresa de propiedad conjunta y de gestión democrática1". Por tanto, podemos obser- 
var que se trata de unas sociedades que no sólo se gestionan y controlan de forma democrática y conjunta, sino que sus fines se amplían a cuestiones de tipo social y cultural, superando los motivos puramente económicos.

Nos preocupa especialmente cómo los directivos de una cooperativa pueden alinear la función social con la función económica y que ello se traduzca en efectos sobre la satisfacción de sus socios, como colectivo por excelencia del movimiento cooperativo así como sobre los restantes grupos de interés. La necesidad de que la cooperativa se oriente al mercado así como la obligación moral de atender una serie de principios y valores que le son específicos, constituyen dos pilares básicos en la gestión cooperativa. Zhao y Gijselinckx (2011) advierten que las cooperativas, como empresas sociales, se encuentran sujetas a un trilema en su actuación determinado por las exigencias del mercado, del estado y de la sociedad civil. Esta cuestión es denominada por los autores anteriores, recogiendo el estudio Côte (2001), como el riesgo de la degeneración de la función social de cooperativas ante la competencia intensa de los mercados.

La propuesta de esta investigación trabaja el equilibrio entre la función económica y social, revisando desde la teoría el caso cooperativo por sus especiales peculiaridades para detectar cómo puede la revelación social mejorar la gestión de este tipo de empresas. En este sentido, el objetivo de este estudio se orienta a analizar cómo la revelación social puede colaborar en el cumplimiento de la función social cooperativa, todo ello sin olvidar la necesaria función económica que desempeñan, ofreciendo una vía para atender las exigencias de RSE que los socios y otros stakeholders de las cooperativas reclaman de éstas.

Spear (2004) concluye que la información de gestión ofrecida por las cooperativas es reducida en términos generales, lo cual choca con el hecho de que la naturaleza cooperativa, que tiene innata una cultura cooperativa de principios y valores de RSE, no aproveche esta situación de ventaja en las relaciones entre ésta y sus diferentes stakeholders (Gallardo et al., 2013). Entendemos que la comunicación es fundamental para poder atender tal ventaja y, especialmente, para ofrecer a sus socios, como grupo de interés estratégico, la información necesaria para su correcta integración en la cooperativa.

La revelación social cooperativa presenta diversas iniciativas que cuentan con un grado considerable de reconocimiento. Tradicionalmente, el Balance Cooperativo ha sido el instrumento que las cooperativas han utilizado para expresar los aspectos sociales y medioambientales aunque con diferentes enfoques y contenidos (Rondot y Bouchard, 2003). Por su parte, el caso español cuenta adicionalmente con una iniciativa específica desarrollada al amparo de cooperativas catalanas, la herramienta RSE.COOP, elaborada por CEPES (2007). Siguiendo a Castilla y Gallardo (2014) podemos observar que, frente al papel preponderante del Balance Cooperativo en años anteriores, nos encontramos en una situación donde éste ha sido sustituido por la metodología Global Reporting Initiative (GRI) y, en un número menor de ocasiones, por la herramienta RSE.COOP. De hecho, disponer de una guía procedente de la iniciativa GRI, y específica para la Economía Social (GRI, 2007), 
que facilita aún más su integración dentro del ámbito cooperativo, unido a su puesto dentro del ranking de herramientas para la revelación social, justifica nuestra decisión de restringir el presente estudio a esta herramienta. Consideramos que esta elección da relevancia al trabajo al tratarse de un estándar internacional, lo cual permitiría la extensión de nuestras conclusiones a otros ámbitos de estudio fuera de nuestras fronteras.

De forma más concreta, todo lo expuesto hasta el momento se traduce en tres cuestiones de investigación más específicas a las que pretendemos dar respuesta a través de la revisión teórica llevada a cabo:

1. Estudiar cómo la revelación puede influir en el establecimiento de unas relaciones más adecuadas en línea con los planteamientos de la Teoría de los Stakeholders.

2. Analizar las implicaciones del papel estratégico del socio cooperativo y de la necesidad de una adecuada comunicación entre socio-cooperativa.

3. Plantear la utilización de la guía GRI de la Economía Social como herramienta de gestión para el caso que nos ocupa.

El trabajo se estructura como sigue: en primer lugar se lleva a cabo un análisis de los grupos de interés para el caso cooperativo, prestando especial atención al papel del socio. A continuación se aborda la importancia de la comunicación corporativa como expresión del compromiso de las cooperativas con la sociedad y haciendo hincapié en la memoria de sostenibilidad con la metodología GRI como una herramienta para la gestión cooperativa. Se finaliza con una reflexión a modo de conclusiones así como planteando las líneas de investigación que emanan de este trabajo.

\section{2.- Los grupos de interés o stakeholders en el caso cooperativo}

Según la Teoría de los Stakeholders la realización de cualquier actividad empresarial ha de ir encaminada a favorecer las relaciones y los intereses de las distintas partes implicadas. Para Atkinson et al. (1997) los grupos de interés se clasifican como del entorno: clientes, accionistas y sociedad, y del proceso: trabajadores y proveedores. En opinión de Frooman (1999) también existen dos grandes grupos, estratégicos y morales, siendo el criterio de la clasificación propuesto de índole estratégico. Así, los primeros se corresponden con colectivos que afectan al funcionamiento de la organización debiendo ser considerados por ésta dentro de su gestión, mientras que los segundos resultan afectados por las acciones empresariales. Otra clasificación relevante es ofrecida por Werther y Chandler (2011) quienes los clasifican en económicos, organizacionales y sociales. Por los primeros se entienden todos aquellos grupos que proceden de la actividad económica empresarial. Los grupos organizacionales 
provienen de relaciones personales dentro de la empresa a diferencia de los sociales quienes representan a todos aquellos colectivos externos que, sin relación económica o personal, se encuentran en el entorno de la empresa.

Dependiendo de las peculiaridades de cada empresa, el número de grupos de interés puede variar cuantitativamente y cualitativamente (Castilla y Gallardo, 2003). Incluso dentro de una misma entidad, los grupos de interés pueden perder o adquirir su condición de estratégicos (Freeman, 1984). Esta circunstancia es significativa hasta el punto en que la empresa tendrá que relajar o aumentar la búsqueda de la satisfacción de cada relación en concreto. Menos habitual resulta que los grupos de interés cambien su condición de internos y externos al igual que también es complicado un trasvase entre las categorías económica, organizacional y social. Por todo ello, resulta esencial la clasificación de los stakeholders por parte de una empresa, ello determinará la trascendencia de cada relación en concreto.

El enfoque de la RSE se centra en el compromiso de la empresa para evitar el daño y para mejorar el bienestar de los stakeholders y de la sociedad en su conjunto (Mohr et al., 2001). Aparece por este motivo una relación clara entre las demandas de los stakeholders en cualquier organización y el papel que la RSE ocupa en ellas. Cada día la gestión socialmente responsable de los negocios se convierte en un requisito implícito en el ámbito empresarial. Birth et al. (2008:184) argumentan que "los objetivos deberían ser definidos en línea con los temas emergentes y los correspondientes stakeholders". Estos temas incluyen la misión, la visión y valores, el clima del lugar de trabajo, el diálogo social, derechos humanos, la participación en la comunidad, el desarrollo de la economía local, el entorno, las relaciones de mercado, y la ética (Birth et al., 2008). De hecho, la última definición ofrecida por la Comisión Europea (2011) al respecto de este concepto utiliza el marco de los stakeholders señalando "la responsabilidad de las empresas por su impacto en la sociedad" y haciendo referencia expresa a la necesidad de colaboración con las partes interesadas para "integrar las preocupaciones sociales, medioambientales y éticas, el respeto de los derechos humanos y las preocupaciones de los consumidores en sus operaciones empresariales y su estrategia básica" (Comisión Europea, 2011:7).

Centrándonos en el caso cooperativo, debemos indicar que la actividad cooperativizada supone respetar una serie de principios y de valores que constituyen la identidad cooperativa (Castilla y Gallardo, 2011a, 2011b), siendo la nota distintiva por excelencia de este tipo de empresas y el nexo fundamental para justificar su RSE. Por otro lado, en el ámbito externo, y como en cualquier otra entidad, las cooperativas necesitan llevar a cabo su actividad principal de una forma rentable, la función económica. En definitiva, y aún cuando existen particularidades unidas a la naturaleza intrínseca de las cooperativas, somos conscientes de que la orientación económica de estas sociedades se relaciona con la viabilidad de conseguir su objetivo dentro del mercado, no observando diferencia en la actividad económica o instrumental de este tipo de sociedades con respecto a cualquier otra fórmula mercantil (Castilla y Gallardo, 2011a, 2011b). 


\section{LA REVELACIÓN SOCIAL A PARTIR DE LA GUÍA GRI DE LA ECONOMÍA SOCIAL: UNA HERRAMIENTA PARA MEJORAR LAS RELACIONES SOCIO-COOPERATIVA}

Si observamos y analizamos la actitud de las cooperativas y los diversos grupos con los que mantienen actuaciones, es evidente que, al igual que indican Belhauri et al. (2005) son precisamente los principios cooperativos los que determinan una serie de conductas que definen la actuación de la cooperativa y su RSE frente a sus stakeholders (socios-propietarios, empleados, clientes, proveedores y comunidad local donde opera). Para Server y Capó (2011) las cooperativas deben aprovechar la ventaja que mantienen frente a la gran empresa en temas de RSE, integrándola en sus políticas de gestión para la búsqueda de sinergias y beneficios mutuos con sus grupos de interés. Marín et al. (2010) relacionan esta cuestión con la creación de valor y la obtención de ventajas competitivas duraderas si se adopta tal tipo de gestión.

Si nos delimitamos al caso de los stakeholders cooperativos, cabe entender en un sentido amplio la participación del socio dentro de una cooperativa, ya que no sólo se refiere a aspectos decisorios o de mera gestión, sino que también abarca la intervención en la actividad productiva, en el capital y en los resultados. Es por este motivo que consideramos que la clasificación entre interno o externo, dependiendo del grupo de interés que se considere, pierde parte de su significado cuando se aplica a este caso concreto, adquiriendo mayor interés la consideración del socio como stakeholder estratégico sea cual fuere el rol o función desempeñada.

Los socios de una cooperativa pueden ser además de propietarios, puesto que aportan el capital social de la cooperativa, clientes y demandar los productos finales que elabora la cooperativa; proveedores y abastecer de factores productivos necesarios a aquélla; empleados y desarrollar diferentes funciones tanto a lo largo de la cadena productiva como directiva de estas empresas ejerciendo labores de gestión. Por tanto, pueden cubrir de manera simultánea diversos roles, todos ellos representativos de los principales stakeholders presentes en cualquier empresa. Prueba de ello son los roles prioritarios ocupados por socios según el tipo de cooperativa que trataremos con posterioridad. De hecho, la identificación del socio como colectivo clave para la investigación del campo cooperativo es una constante que se repite en un elevado número de estudios, entre ellos, el trabajo de revisión de Marín et al. (2010) analiza el papel asignado al socio dentro de los diferentes marcos teóricos utilizados para estudiar el gobierno de las empresas cooperativas.

De cualquier definición de sociedad cooperativa que analicemos se desprende que la participación del socio dentro de esta institución requiere su implicación personal. Incluso cabe afirmar que es imprescindible la capacidad para participar personalmente en el objeto social de la cooperativa, junto a otros determinados requisitos de carácter societario, representando la pérdida de dicha capacidad, de forma general, un motivo de baja obligatoria. De forma más concreta, y siguiendo a GarcíaGutiérrez (1989:104 y ss.) cabe diferenciar tres tipos de flujos de participación: a) Reales, a través del desarrollo de la actividad productiva como proveedores de materiales y/o trabajo y como consumidores, b) Financieros, como prestamistas aportando financiación a la cooperativa tanto en el momento inicial como en su caso en posteriores y c) Informativo-decisionales, se les supone desde la teoría a participar en la dirección y gestión de la cooperativa a través del principio de democracia de una persona un voto. Este triple rol del socio en la cooperativa también ha sido recogido con posterioridad por 
Hernández (2001) quien lo aplica al caso de las cooperativas agrícolas, por Pérez-Fernández (2002) en el ámbito más general de la economía social y por Vargas y Vacas (2005) quienes lo aplican al ámbito específico de la RSE.

Los socios prestan atención a aspectos económicos tales como el precio de los productos, la calidad y el servicio, pero también se preocupan por la capacidad que tiene la cooperativa para integrar la "diferencia cooperativa" en todos los aspectos del negocio (Brown y Hicks, 2007). No se entiende el éxito de la cooperativa sin atender a sus objetivos sociales. Es por ello que, siguiendo a Genovart (2012), resulte necesario atender dicho carácter social.

\subsection{Tipos de cooperativas y roles socio-cooperativa}

Una vez que se ha presentado el papel del socio y su integración bajo diferentes roles dentro de la cooperativa, pasamos a analizar con mayor profundidad esta cuestión. Para ello, nos atenemos a la Ley 27/1999, de 16 de julio, de Cooperativas (LGC) por la que se regula esta forma jurídica en el caso español y donde en su artículo $6.1^{2}$ las cooperativas se clasifican en las siguientes categorías. En la tabla $n^{\circ} 1$ se puede observar el detalle según esta ley de las diferentes categorías existentes en función de su actividad básica o fundamental así como el papel fundamental que desempeña el socio en cada una de ellas.

\section{Tabla 1. Clasificación de cooperativas según la LGC3 y el rol fundamental del socio}

\begin{tabular}{|l|l|l|}
\hline Tipo & Descripción & $\begin{array}{l}\text { Papel fundamental } \\
\text { del socio }\end{array}$ \\
\hline $\begin{array}{l}\text { Cooperativas de } \\
\text { trabajo asociado }\end{array}$ & $\begin{array}{l}\text { "Son cooperativas de trabajo asociado las que tienen por objeto proporcionar a sus } \\
\text { socios puestos de trabajo, mediante su esfuerzo personal y directo, a tiempo par- } \\
\text { cial o completo, a través de la organización en común de la producción de bienes } \\
\text { o servicios para terceros". }\end{array}$ & Socio-trabajador \\
\hline $\begin{array}{l}\text { Cooperativas de } \\
\text { consumidores y } \\
\text { usuarios }\end{array}$ & $\begin{array}{l}\text { "Son cooperativas de consumidores y usuarios aquéllas que tienen por objeto el } \\
\text { suministro de bienes y servicios adquiridos a terceros o producidos por sí mismas, } \\
\text { para uso o consumo de los socios y de quienes con ellos conviven, así como la } \\
\text { educación, formación y defensa de los derechos de sus socios en particular y de } \\
\text { los consumidores y usuarios en general". }\end{array}$ & Socio-cliente \\
\hline $\begin{array}{l}\text { Cooperativas de } \\
\text { viviendas }\end{array}$ & $\begin{array}{l}\text { "Las cooperativas de viviendas asocian a personas físicas que precisen alojamiento } \\
\text { y/o locales para sí y las personas que con ellas convivan. También podrán ser } \\
\text { socios los entes públicos y las entidades sin ánimo de lucro, que precisen aloja- } \\
\text { miento para aquellas personas que dependientes de ellos tengan que residir, por } \\
\text { razón de su trabajo o función, en el entorno de una promoción cooperativa o que } \\
\text { precisen locales para desarrollar sus actividades". }\end{array}$ & Socio-cliente \\
\hline
\end{tabular}

2.- Artículo 6 redactado por el número uno de la disposición final segunda de la Ley 13/2013, 2 agosto, de fomento de la integración de cooperativas y de otras entidades asociativas de carácter agroalimentario (B.O.E. 3 agosto).

3.- No se han considerado las cooperativas de crédito por resultar un tipo de cooperativas para las cuales se aclara que se regirán por su ley específica y por sus normas de desarrollo.

CIRIEC-España, Revista de Economía Pública, Social y Cooperativa

№ 83/2015, pp. 143-168

ISSN: 0213-8093 


\begin{tabular}{|c|c|c|}
\hline $\begin{array}{l}\text { Cooperativas } \\
\text { agroalimentarias }\end{array}$ & $\begin{array}{l}\text { "Son cooperativas agroalimentarias las que asocien a titulares de explotaciones } \\
\text { agrícolas, ganaderas o forestales, incluyendo a las personas titulares de estas } \\
\text { explotaciones en régimen de titularidad compartida, que tengan como objeto la } \\
\text { realización de todo tipo de actividades y operaciones encaminadas al mejor apro- } \\
\text { vechamiento de las explotaciones de sus socios, de sus elementos o componen- } \\
\text { tes de la cooperativa y a la mejora de la población agraria y del desarrollo del } \\
\text { mundo rural, así como a atender a cualquier otro fin o servicio que sea propio de } \\
\text { la actividad agraria, ganadera, forestal o estén directamente relacionados con ellas } \\
\text { y con su implantación o actuación en el medio rural". }\end{array}$ & Socio-proveedor \\
\hline $\begin{array}{l}\text { Cooperativas } \\
\text { de explotación } \\
\text { comunitaria de } \\
\text { la tierra }\end{array}$ & $\begin{array}{l}\text { "Son cooperativas de explotación comunitaria de la tierra las que asocian a titula- } \\
\text { res de derechos de uso y aprovechamiento de tierras u otros bienes inmuebles, } \\
\text { susceptibles de explotación agraria, que ceden dichos derechos a la cooperativa } \\
\text { y que prestan o no su trabajo en la misma, pudiendo asociar también a otras per- } \\
\text { sonas físicas que, sin ceder a la cooperativa derechos de disfrute sobre bienes, } \\
\text { prestan su trabajo en la misma, para la explotación en común de los bienes cedi- } \\
\text { dos por los socios y de los demás que posea la cooperativa por cualquier título". }\end{array}$ & Socio-trabajador \\
\hline $\begin{array}{l}\text { Cooperativas } \\
\text { de servicios }\end{array}$ & $\begin{array}{l}\text { "Son cooperativas de servicios las que asocian a personas físicas o jurídicas, titu- } \\
\text { lares de explotaciones industriales o de servicios y a profesionales o artistas que } \\
\text { ejerzan su actividad por cuenta propia, y tienen por objeto la prestación de sumi- } \\
\text { nistros y servicios, o la producción de bienes y la realización de operaciones enca- } \\
\text { minadas al mejoramiento económico y técnico de las actividades profesionales } \\
\text { o de las explotaciones de sus socios". }\end{array}$ & Socio-proveedor \\
\hline $\begin{array}{l}\text { Cooperativas } \\
\text { del mar }\end{array}$ & $\begin{array}{l}\text { "Son cooperativas del mar las que asocian a pescadores, armadores de embar- } \\
\text { caciones, cofradías, organizaciones de productores pesqueros, titulares de vive- } \\
\text { ros de algas, de cetáreas, mariscadores y familias marisqueras, concesionarios } \\
\text { de explotaciones de pesca y de acuicultura y, en general, a personas físicas o jurí- } \\
\text { dicas titulares de explotaciones dedicadas a actividades pesqueras o de indus- } \\
\text { trias marítimo-pesqueras y derivadas, en sus diferentes modalidades del mar, rías } \\
\text { y lagunas marinas, y a profesionales por cuenta propia de dichas actividades, y } \\
\text { tienen por objeto la prestación de suministros y servicios y la realización de ope- } \\
\text { raciones, encaminadas al mejoramiento económico y técnico de las actividades } \\
\text { profesionales o de las explotaciones de sus socios". }\end{array}$ & Socio-proveedor \\
\hline $\begin{array}{l}\text { Cooperativas } \\
\text { de transportistas }\end{array}$ & $\begin{array}{l}\text { "Son cooperativas de transportistas las que asocian a personas físicas o jurídicas, } \\
\text { titulares de empresas del transporte o profesionales que puedan ejercer en cual- } \\
\text { quier ámbito, incluso el local, la actividad de transportistas, de personas o cosas } \\
\text { o mixto, y tienen por objeto la prestación de servicios y suministros y la realiza- } \\
\text { ción de operaciones, encaminadas al mejoramiento económico y técnico de las } \\
\text { explotaciones de sus socios". }\end{array}$ & Socio-proveedor \\
\hline $\begin{array}{l}\text { Cooperativas } \\
\text { de seguros }\end{array}$ & $\begin{array}{l}\text { "Son cooperativas de seguros las que ejerzan la actividad aseguradora, en los } \\
\text { ramos y con los requisitos establecidos en la legislación del seguro y, con carác- } \\
\text { ter supletorio, por la Ley de Cooperativas". }\end{array}$ & Socio-cliente \\
\hline $\begin{array}{l}\text { Cooperativas } \\
\text { sanitarias }\end{array}$ & $\begin{array}{l}\text { "Son cooperativas sanitarias las que desarrollan su actividad en el área de la salud, } \\
\text { pudiendo estar constituidas por los prestadores de la asistencia sanitaria, por los } \\
\text { destinatarios de la misma o por unos y otros. Podrán realizar también actividades } \\
\text { complementarias y conexas incluso de tipo preventivo, general o para grupos o } \\
\text { colectivos determinados". }\end{array}$ & $\begin{array}{l}\text { Socio-trabajador/socio- } \\
\text { cliente }\end{array}$ \\
\hline $\begin{array}{l}\text { Cooperativas } \\
\text { de enseñanza }\end{array}$ & $\begin{array}{l}\text { "Son cooperativas de enseñanza las que desarrollan actividades docentes, en sus } \\
\text { distintos niveles y modalidades. Podrán realizar también, como complementarias, } \\
\text { actividades extraescolares y conexas, así como prestar servicios que faciliten las } \\
\text { actividades docentes". }\end{array}$ & Socio-trabajador \\
\hline
\end{tabular}

FUENTE: Elaborado a partir de la LGC (1999).

CIRIEC-España, Revista de Economía Pública, Social y Cooperativa 
Como se puede observar, la superposición del socio en diferentes actividades económicas es un hecho fundamental a considerar en la gestión cooperativa. Es por este motivo que su condición de grupo de interés estratégico nos lleva a profundizar en el conocimiento de cada una de estas funciones en los apartados siguientes. Obviamente, dependiendo del tipo de categoría en la que nos encontremos el valor estratégico del socio dependerá del rol desempeñado, así mientras en cooperativas de consumidores la importancia del socio depende tanto de esta condición como de su papel de cliente, esto difiere de otros tipos de cooperativas, a modo de ejemplo de las cooperativas agroalimentarias donde su relevancia procede de su función de proveedor además de su papel de socio. Esto es, dependiendo del tipo de cooperativa el rol estratégico del socio debe ser analizado para la gestión correcta de tal relación en aplicación de la Teoría de los Stakeholders.

A este respecto, y siguiendo a Mozas et al. (1997:100), debemos indicar que el poseer un determinado rol no es un hecho excluyente. De hecho, la combinación de roles acarrea el problema de que en función de las circunstancias de cada uno de ellos, los socios, un rol puede suponer más que otro "en la mente del cooperativista", produciéndose una jerarquización de los roles a desempeñar. Esta circunstancia viene a incidir directamente en el necesario análisis por parte de la cooperativa de sus socios y sus intereses para una adecuada relación socio-cooperativa.

\subsection{El rol del socio-proveedor}

Los proveedores, como en cualquier otra empresa, desempeñan en la cooperativa una función fundamental en la entrega de materiales y materias necesarias para la fabricación. El compromiso de los socios proveedores puede ser entendido como el deseo de permanecer en una cooperativa y no dejarla ante ofertas de mejores precios y/o servicios, tal y como señalan Cechin et al. (2013). Para estos autores, el compromiso resulta de una combinación de incentivos de mercado, de control jerárquico, de compromiso con la comunidad, democracia y de comunicación.

Cuando nos encontramos ante empresas cooperativas donde los proveedores son socios, tal suministro presenta unas notas distintivas que pasamos a comentar. Por un lado, debemos indicar las consecuencias positivas que para las cooperativas representa la posesión de proveedores fijos. Éstas son de diversa índole como la garantía de estabilidad en el aprovisionamiento, el ahorro de costes y tiempo en la búsqueda de materias primas y la facilidad en el contacto.

Por otro lado, pueden aparecer también problemas. Para Aragonés (1987) la primacía del rol de proveedor provoca que el cooperativista tenga como objetivo fundamental el maximizar el precio de los productos que entrega, siendo por este motivo partidario de una visión a corto plazo. La obligación de aceptar todas las entregas -con mayores o menores calidades- supone el principal inconveniente de la identificación socio-proveedor. Caballer (1992) añade dos perjuicios más: la reducción de las rentas derivadas de la dimensión y las dificultades inherentes a la planificación por la supeditación de la producción cooperativa a la producción entregada por los socios. Para Reguant (2012), cuando el 


\section{LA REVELACIÓN SOCIAL A PARTIR DE LA GUÍA GRI DE LA ECONOMÍA SOCIAL: UNA HERRAMIENTA PARA MEJORAR LAS RELACIONES SOCIO-COOPERATIVA}

proveedor es propietario, la capacidad de negociación para la cooperativa es nula, lo que afecta a su competitividad. Siguiendo a Bijman (2009) y Hanf (2009) en entornos donde la calidad final del producto es fundamental, puede ser que las cooperativas estén forzadas a coordinarse con sus socios proveedores para poder cumplir tales requisitos. Sólo el control más estricto puede asegurar que tal cuestión no sea un problema (Hanf, 2009), si bien, no es ésta una cuestión sencilla. Según Nilsson et al. (2012) las estrategias de coordinación vertical que exigen controlar a los socios tienen costes invisibles que pueden afectar negativamente al compromiso de los socios y, por extensión, al desempeño cooperativo.

\subsection{El rol del socio-cliente}

Abordamos en este apartado el análisis de las peculiaridades que presenta la función de comercialización dentro de sociedades cooperativas, labor que llevaremos a cabo desde el solapamiento de la figura del socio con esta función. Las ventajas de la identificación de socios y clientes son amplias, entre ellas, la de eliminar prácticamente las acciones comerciales al quedar asegurada la venta. Las cooperativas de consumo como ejemplos límite de tal solapamiento presentan por tales motivos una problemática comercial totalmente diferente a la de la empresa capitalista.

Si bien, y aunque el caso cooperativo presente una casuística singular, la satisfacción de los clientes aunque sean socios también resulta fundamental, ¿qué sucede con su satisfacción? Ahora la satisfacción se encuentra condicionada tanto con el producto/servicio como con la función social desempeñada por la cooperativa. Lo ideal sería que ambas cuestiones fuesen atendidas por la cooperativa, pues como en cualquier empresa las prácticas sociales o de RSE tienen una influencia positiva en la percepción de sus clientes, incluso Lichtenstein et al. (2004) plantean que la RSE genera identificación de los clientes con la empresa, siendo uno de sus principales beneficios.

No obstante, la percepción que los clientes mantienen sobre la actuación responsable de una empresa tiene consecuencias directas sobre la valoración que le otorgan en términos generales, tal y como señalan Aaijaz et al. (2012). De hecho, siguiendo a estos autores podemos indicar que la cuestión de cómo la RSE afecta a la satisfacción de clientes y otros grupos de interés se ha convertido en uno de los principales temas de interés en los que convergen la investigación en marketing y RSE. No es sólo una cuestión cooperativa, pues como se ha indicado dentro de los beneficios de la actuación responsable estudiados por la literatura se encuentra precisamente la mejora del reconocimiento empresarial o reputación empresarial. Si los clientes consideran que los productos cumplen sus expectativas estarán satisfechos y, qué duda cabe, que la RSE influirá en tal expectativa. 


\subsection{El rol del socio-empleado}

La legislación cooperativa vigente establece diversas formas de participación. Concretamente, la LGC reconoce las figuras de los socios de trabajo y de los colaboradores. Se denominan socios de trabajo a aquellos que prestan su trabajo personal dentro de la cooperativa siempre que no sean de trabajo asociado o de explotación comunitaria de la tierra y en las de segundo grado. Su actividad deberá estar habilitada dentro de los estatutos de las cooperativas de primer grado al igual que también vendrá expresada la relación entre éstos y los demás socios en cuanto a derechos y obligaciones sociales. También la LGC prevé que puedan formar parte de las cooperativas personas físicas o jurídicas que colaboren en la realización de actividades accesorias a la principal, los socios colaboradores. A estos efectos, los estatutos de las entidades en las que se produzca tal participación deberán reflejar debidamente diferenciada la calificación de cada una de las actividades que lleven a cabo.

La doble condición socio-trabajador significa en la práctica que los socios se contratan como trabajadores de la cooperativa. En este sentido, pueden observarse problemas de ambigüedad, pues siguiendo a García-Gutiérrez (1989) esta condición implica derechos y obligaciones contrapuestas para ellos pues tratarán como socio de conseguir la máxima productividad para la cooperativa pero a la vez también se preocuparán por obtener los máximos derechos sociales por cuanto su papel de trabajadores.

\subsection{El rol del socio-directivo}

Para Mozas et al. (1997) los socios son empresarios por cuanto llevan a cabo una actividad individualmente y se unen para realizar otras conjuntamente, decidiendo el futuro de la sociedad que comparten. Éstas serían las recomendaciones teóricas de la gestión según los principios y valores cooperativos establecidos por la $\mathrm{ACl}$ (1995). Por otro lado, podemos indicar que resultan varios los motivos que catalogan como "especial" la gestión cooperativa. Así, mientras en cualquier tipo de sociedad los directivos tienen la posibilidad de adecuar la estructura organizativa a las peculiaridades de su actividad de la forma que estimen más conveniente, esto no ocurre en una cooperativa al existir una legislación específica sobre la obligación de crear una determinada estructura social "democrática constituida por todos los socios que a través de los canales establecidos eligen a sus representantes" (Mozas, 1997:144).

Hueth y Marcoul (2009) diferencian la gestión privada o capitalista como aquella responsable de la obtención de máximos resultados para inversores de la gestión cooperativa como la tendente a dar máximos resultados a socios, siendo lo último una cuestión diferenciadamente más completa que la primera. Si bien, siguiendo a Moyano et al. (2008) lo anterior no implica desatender los objetivos de eficacia y eficiencia dentro de estas empresas. Feng y Hendrikse (2012) plantean dos preocupaciones de los socios, las de propietarios y las de usuarios, dependiendo del tipo de cooperativa en la que 


\section{LA REVELACIÓN SOCIAL A PARTIR DE LA GUÍA GRI DE LA ECONOMÍA SOCIAL: UNA HERRAMIENTA PARA MEJORAR LAS RELACIONES SOCIO-COOPERATIVA}

se integren sea cual sea su tipología. Para Liang y Hendrikse (2013) la gestión de una empresa cooperativa no sólo debe contemplar intereses económicos sino también las expectativas que van más allá. Todo lo anterior redunda en implicaciones tanto para la gestión cooperativa como para los socios que asuman su llevanza en la misma, esto es, el rol socio-directivo.

El Consejo Rector es el órgano de gobierno, gestión y representación de la sociedad cooperativa. Dentro de su composición deben aparecer, al menos, las figuras del presidente -que también lo será de la cooperativa, vicepresidente y secretario. Salvo para el secretario, será imprescindible la calificación de socio en tal ejercicio, produciéndose la superposición entre la condición de socio, propietario y las labores de gestión. Ahora bien, este solapamiento no sólo se manifiesta entre los miembros del Consejo Rector sino que abarca a todos los socios, ya que por definición cualquier cooperativa debe conseguir su fin último mediante la gestión conjunta y democrática de todos sus integrantes. La Asamblea General es precisamente el órgano que agrupa a todos los socios, representando la expresión de la voluntad social. Chaves y Schedwy (2004) plantean que este tipo de gestión democrática, desde la teoría, asegura que los intereses de los socios resulten fundamentales en la gestión cooperativa.

El liderazgo del Consejo Rector corresponde a su presidente, quien a la vez desempeñará este mismo cargo para la cooperativa. La importancia de esta función de dirección exige que aquella persona que se ocupe de la misma posea, como mínimo, ciertos requisitos. Ante la complejidad del entorno actual en el que se desenvuelven las organizaciones y las continuas amenazas y oportunidades que pueden acecharlas, resulta totalmente imprescindible la posesión de un conocimiento profundo y completo del mercado por parte de los presidentes para que éstos puedan desempeñar su actuación de forma correcta. Pero ¿qué ocurre cuando tales conocimientos no se encuentran presentes en la figura del presidente democráticamente electo? La solución al problema de gestión de sociedades cooperativas se relaciona con el hecho de que los estatutos contemplen la creación de una dirección profesionalizada dando lugar a una estructura dualista en la que se podrían salvar los inconvenientes anteriores.

Trechter et al. (2002) encuentran que los socios que han participado en la gestión como directivos o miembros de algún comité así como aquellos otros que han recibido formación cooperativa tienden a estar más comprometidos con su cooperativa. Para Server y Capó (2011) la existencia de sociosdirectivos precisamente asegura que sus intereses se incluyan en el proceso de toma de decisiones, pero no sólo éstos sino también los de aquellos grupos de interés con los que la sociedad interactúa, sus stakeholders. Por su parte, también Liang y Hendrikse (2013) observan divergencias entre los desempeños obtenidos por cooperativas con directivos socios y directivos no socios que quedan condicionadas por terceras variables. 


\section{3.- La comunicación social como vía de compromiso cooperativo}

En el art. 16 de la LGC se regulan los derechos de los socios. En concreto, en el artículo 16.3, se reconoce el derecho de información de los socios y de forma expresa se indica su derecho a recibir, como mínimo, información sobre los asuntos que deban ser tratados en las Asambleas Generales, a recibir una copia de los estatutos, y una copia del Reglamento de régimen interno y de sus modificaciones, si fuera el caso. Sin embargo, ni se indica qué formato debe tener el acceso a esta información ni se regula el derecho a la información relativa a otros ámbitos no estrictamente societarios.

Esta reglamentación de mínimos no es contraria al reconocimiento de que un elemento clave de éxito en la comunicación corporativa de toda organización es su preocupación por tener una relación bilateral significativa, positiva y provechosa con los distintos grupos de stakeholders (Balmer y Greyser, 2006; Galetzka et al., 2008). Y es que "para la mayoría de las empresas, la pregunta no es si hay que comunicarse sino más bien qué decir, a quién, y con qué frecuencia" (Kotler, 2003: 563). El hecho de que una empresa envíe un mensaje a un amplio grupo de stakeholders informando de lo que hace, lo que quiere, cuáles son sus objetivos, es un ejemplo de comunicación integrada corporativa (Arnold, 1993; Best, 2004). Así, la comunicación supera al legítimo derecho a la información y está considerada una parte importante de la gestión que asegura el futuro del negocio (Charter y Polonsky, 1999; Mark-Herbert y von Schantz, 2007). Esto es así hasta el punto de que se la considera responsable de la reputación empresarial en la medida en que ésta se consigue a través de la capacidad de ofrecer información a cada uno de los grupos de interés sobre la empresa y sobre la capacidad que tiene a su vez de atender sus expectativas (De los Rios et al., 2012).

Por tanto, no cabe duda de que la comunicación es fundamental en cualquier organización, incluida la de tipo cooperativo. En este caso concreto, la necesidad de aprovechar la comunicación como herramienta estratégica es, si cabe, todavía más relevante y justifica nuestra propuesta. Siguiendo a Maignan y Ferrell (2004) las empresas deben diseñar sus estrategias de comunicación sabiendo cómo van a reaccionar sus grupos de interés para intentar atenderlos. Especialmente, en el caso cooperativo creemos, ante los solapamientos existentes entre diversas realidades económicas que se acogen a la forma cooperativa y el papel multifuncional de los socios, que el proceso de comunicación resulta fundamental por su valor como vía para que los socios evalúen y juzguen las actuaciones empresariales en su conjunto, debiendo ser considerados como parte esencial. Pérez-Fernández (2002) establece que una correcta comunicación en las empresas de la economía social como las cooperativas contribuye a reducir situaciones negativas como envidias, desconfianzas, y tensiones entre los socios al tiempo que se potencian aquellas situaciones positivas como son el buen clima social y el compañerismo, entre otros. 


\section{LA REVELACIÓN SOCIAL A PARTIR DE LA GUÍA GRI DE LA ECONOMÍA SOCIAL: UNA HERRAMIENTA PARA MEJORAR LAS RELACIONES SOCIO-COOPERATIVA}

Mozas et al. (2010) concluyen que, a pesar de la facilidad que las empresas cooperativas podrían tener para ejercer y transmitir la RSE, no se visualizan las acciones a través de sus páginas web, siendo éste uno de los medios más cómodos para la revelación voluntaria de cualquier tipo de empresa. Server y Capó (2011) consideran que los informes de sostenibilidad son claves a este respecto, cuestión a la que nos adherimos presentando la justificación teórica correspondiente.

\subsection{Un paso más en la comunicación: el empleo de estándares internacionales}

Aunque, tal y como se ha indicado, las cooperativas no constituyen ejemplos en el ámbito de la revelación social, en los últimos años parece observarse un creciente interés en la utilización de estándares internacionales de RSE (Castilla y Gallardo, 2013). El interés apuntado ha desencadenado en una versión específica para el caso español de la GRI G3 denominada "Ciclo preparatorio para la elaboración de memorias de sostenibilidad GRI: manual para organizaciones pequeñas y medianas. Edición especial para la Economía Social", de plena aplicación para cooperativas y a la que nos venimos refiriendo.

La GRI (2007: 29) se refiere explícitamente a un proceso de identificación de stakeholders amplio bajo los siguientes cinco aspectos:

a) Responsabilidad: entendiendo por ésta los grupos vinculados en función de normativas, contratos y/o políticas legales, económicas y operativas

b) Influencia: Ios que afectan a la consecución de los objetivos tanto con influencia informal como formal interna y externa

c) Proximidad: grupos de dependencia diaria y de cercanía física a la organización

d) Dependencia: los que resulten básicos para la organización, clientes y proveedores

e) Representación: las instituciones clave con las que se relaciona la organización, sindicatos, líderes de la comunidad, políticos, entre otros

Así, tras su identificación, la GRI (2007) propone su clasificación, como estratégicos o no, y el diálogo con ellos con vistas a mejorar la utilidad de esta herramienta. Concretamente, el papel del socio en los criterios para su selección como colectivo resulta especialmente evidente para el apartado relativo a influencia, proximidad y dependencia, por cuanto todos estos atributos se cumplen para este colectivo de forma innata, esto es, por su condición de socios.

De forma más específica, el manual posibilita que una organización de la Economía Social pueda dar cuenta de su desempeño como cualquier otra empresa utilizando los indicadores GRI e indicando igualmente su relación con los valores y principios de la Economía social, mediante indicadores específicos $^{4}$, así como poder informar de otros indicadores concretos de su propia actividad. La forma ope-

4.- Para una mayor información puede consultarse la Tabla de Indicadores de la Economía Social y correspondencia con el Global Reporting Initiative. Disponible en: http://www.cooperativescatalunya.coop/attachments/054_indicadores_economia_social.pdf. 
rativa de vincular los principios de la Economía Social con la GRI es estableciendo una serie de indicadores para cada uno de los principios de la Economía Social así como la correspondencia existente entre ambos. Con esto se consigue, por un lado mantener la especificidad de la cooperativa en el caso concreto que nos ocupa, así como mantener la visión comparativa a nivel internacional que surge de la consideración de la GRI como estándar internacional de RSE. De hecho, la trascendencia de la utilización de este estándar puede corroborarse dentro del estudio realizado por KMPG (2013) al indicar el mismo que el $78 \%$ de las empresas que están revelando información social lo hacen al amparo de la metodología GRI, situándose este porcentaje en un $82 \%$ para el caso de las 250 empresas más grandes del mundo.

\section{Tabla 2. Detalle de la GRI (2007)}

\begin{tabular}{|c|c|}
\hline Principios de Economía Social & Dimensiones de la RSE de referencia \\
\hline I. Primacía de las personas y del objeto social sobre el capital & - Importancia del objetivo social \\
\hline II. Adhesión voluntaria y abierta & $\begin{array}{l}\text { - Exigencias para la accesibilidad de propietarios en un } \\
\text { sentido amplio: incorporación y salida }\end{array}$ \\
\hline $\begin{array}{l}\text { III. Organización y cultura empresarial con vocación de gestión } \\
\text { participativa y democrática }\end{array}$ & $\begin{array}{l}\text { - Gobierno democrático } \\
\text { - Formación } \\
\text { - Información }\end{array}$ \\
\hline $\begin{array}{l}\text { IV. Conjunción de los intereses de los miembros usuarios y } \\
\text { del interés general }\end{array}$ & $\begin{array}{l}\text { - Grupos de interés } \\
\text { - Derechos humanos } \\
\text { - Identidad cultural }\end{array}$ \\
\hline V. Defensa y aplicación de los principios de solidaridad & $\begin{array}{l}\text { - Actuación en la sociedad } \\
\text { - Actuación en el medio ambiente } \\
\text { - Gestión de grupos de interés }\end{array}$ \\
\hline $\begin{array}{l}\text { Vl. Autonomía de gestión e independencia respecto } \\
\text { de los poderes públicos }\end{array}$ & - Gestión democrática \\
\hline $\begin{array}{l}\text { VII. Aplicación de los excedentes o de la mayor parte de los } \\
\text { mismos en la consecución de los objetivos a favor del interés } \\
\text { general, de los servicios a los miembros }\end{array}$ & $\begin{array}{l}\text { - Reparto de beneficios responsable } \\
\text { - Reparto de beneficios social } \\
\text { - Reparto de beneficios equitativo }\end{array}$ \\
\hline
\end{tabular}

FUENTE: Adaptado de Castilla y Gallardo (2014).

Si analizamos el caso concreto del principio "conjunción de los intereses de los miembros usuarios y del interés general" -véase tabla $n^{0} 3$-, podemos observar que la Teoría de los Stakeholders aparece presente en los fundamentos de este modelo de memoria de sostenibilidad, lo cual evidencia nuestra apuesta por su selección al entender la trascendencia de esta teoría para la gestión cooperativa. 


\section{Tabla 3. Indicadores de la Economía Social para el Cuarto Principio}

\begin{tabular}{|l|l|}
\hline Principio & Detalle \\
\hline IV: Conjunción de los intereses de los & Definición de un mapa de los grupos de interés focalizados en la organización \\
miembros usuarios y del interés general & Flujos relacionales existentes entre la organización y sus grupos de interés \\
& Existencia de un apartado sobre derechos humanos dentro de la política de RSE \\
& Adaptación a la identidad cultural de los territorios donde actúa la organización \\
& Existencia de una valoración de la satisfacción de los grupos de interés \\
\hline
\end{tabular}

FUENTE: GRI (2007:46).

Al hilo de lo anterior, Reinhard et al. (2010) se refieren a la comunicación en un doble sentido: cuando es simplemente empleada para compartir información y cuando está orientada al diálogo. En el primer caso, se hace referencia a una información que solo necesita ser transmitida a ciertos stakeholders (Morsing y Schultz, 2006) pero no se requiere una retroalimentación sobre ella, mostrando aquéllos un rol pasivo. Sin embargo, en el segundo caso, la comunicación conlleva la apertura de un diálogo y la existencia, por tanto, de una retroalimentación entre las partes, siendo este tipo el que permitiría conjugar los intereses de todos los miembros de la cooperativa o, al menos, conocerlos para tratar de llegar a un acercamiento entre ellos. En este caso, los stakeholders juegan un papel activo a partir de las respuestas y reacciones ante quien inició la comunicación (Reinhard et al., 2010).

\section{4.- La comunicación social y transparencia en las cooperativas}

Relacionado con la comunicación se encuentra la propia transparencia de la organización cooperativa. En general, la transparencia es entendida como un valor empresarial apreciado por los stakeholders, máxime en el contexto de escándalos financieros, fraude y corrupción con el que se ha iniciado el tercer milenio (Ramonet, 2001). Siguiendo al conocido Informe Baldassarre (UE, 2012), la actual crisis en la que nos encontramos tiene entre sus orígenes los errores cometidos en materia de transparencia, rendición de cuentas, responsabilidad y en una visión a corto plazo de las empresas. De ahí, la oportunidad de considerar la difusión de información social y medioambiental por parte de las empresas en general y de las cooperativas en particular como modo de mejorar la información poseída y divulgada y aprovechar su contribución en la mejora de decisiones empresariales.

Se puede decir por tanto que la transparencia es fundamental para conseguir un doble objetivo: por una parte, el conocimiento por parte de los grupos de interés sobre el comportamiento responsable de una empresa, y por otro lado, como medio de que tal conocimiento mejore las relaciones con 
los stakeholders y permita la obtención de beneficios estratégicos para la misma, tal y como lo mantiene la Teoría de los Stakeholders. Igualmente, la doble concepción de la actividad cooperativa (económica y social) justifica el empleo de la comunicación para completar el conocimiento de la función económica 0 actividad de explotación, que se traduce en la elaboración de información contable básica como en cualquier otra, con la decisión voluntaria de completar la comunicación cooperativa con información específica a los temas de índole social que redundan en la justificación de la función social realizada por cualquier cooperativa.

Hasta ahora, los estudios de los niveles de transparencia para la prevención de la corrupción se habían focalizado en el análisis del sector público definiendo la corrupción como la utilización del cargo público en interés propio de los funcionarios. Pero la organización Transparencia Internacional en España ha desarrollado unos principios para las empresas donde se manifiesta la necesidad de que éstas adopten medidas que favorezcan la transparencia y que, lejos de verse consideradas como potenciales autoras de conductas de corrupción, tengan una adecuada protección interna contra la misma, también para sus directivos y para todos los miembros de la estructura empresarial (Transparencia Internacional, 2013).

En este contexto de apuesta por la transparencia empresarial, y siguiendo la Guía GRI (2007:3) podemos afirmar que las cooperativas, como empresas de Economía Social, muestran un elevado interés en la transparencia, rendición de cuentas y elaboración de memorias de sostenibilidad, al señalarse que "la economía social es la primera beneficiada por el hecho de dar a conocer sus especiales características y su forma de gestionar riesgos y oportunidades". La elaboración de la memoria de sostenibilidad según la GRI por parte de las cooperativas estaría abordando y asumiendo una función más amplia que la de informar y que la de comunicar. La memoria estaría actuando también como un instrumento al servicio de la transparencia, fundamentalmente de cara a los socios, alejando así cualquier hipótesis de mal gobierno o corrupción.

De manera más específica, consideramos que la finalidad de la revelación social para el caso de las sociedades cooperativas se relaciona con los siguientes elementos:

- Poner de manifiesto la identidad cooperativa

- Ofrecer información sobre la responsabilidad social cooperativa, tanto asumida como ejercida por la cooperativa

- Favorecer la planificación, el proceso presupuestario y el control social tanto a nivel micro como macroeconómico

- Difundir los beneficios de la cooperación

Si bien, no constituyen las cooperativas ejemplos destacables en comunicar su actuación social (Castilla y Gallardo, 2013), hecho que les lleva a perder una serie de ventajas estratégicas, entre ellas las relativas a la posibilidad de mejorar las relaciones con sus stakeholders a través de estas entregas, cuestión fundamental para este trabajo. Mugarra (2001:38) al respecto indica que las diferentes 


\section{LA REVELACIÓN SOCIAL A PARTIR DE LA GUÍA GRI DE LA ECONOMÍA SOCIAL: UNA HERRAMIENTA PARA MEJORAR LAS RELACIONES SOCIO-COOPERATIVA}

iniciativas desde el mundo cooperativo para demostrar su interés por la RSE no han sido todo lo importantes que debieran, mereciendo destacar el caso de la Confédération Générale des Sociétés Coopératives Ouvriéres de Production (SCOP) francesas que ya desde 1965 comienza a elaborar un Balance Cooperativo. También encontramos ejemplos posteriores donde sobresale la preocupación por el cooperativismo de consumo ya que "parecen ser las más sensibilizadas al respecto y las que más fuerte están apostando por la implantación y mantenimiento de esta vía de información y gestión social en sus cooperativas" (Mugarra, 2001:44). Recientemente, Monzón y Antuñano (2012), tras el análisis de una veintena de casos relevantes, concluyen afirmando que la RSE se encuentra bastante desarrollada en el sector cooperativo español, si bien, con diferentes formas de entenderla y aplicarla por este tipo de entidades. Pero sin lugar a dudas, entendemos que la tarea pendiente es la revelación social cooperativa.

Si traducimos todo lo analizado hasta este momento al caso cooperativo, podemos observar tres cuestiones realmente relevantes:

a) El papel del socio y la necesidad de buscar su compromiso con la cooperativa tanto para el cumplimiento de la función social atribuida como para la actividad económica llevada a cabo en la misma. Esto es, su concepción como stakeholder estratégico.

b) La necesidad de que una correcta comunicación aborde el conocimiento más profundo de las decisiones cooperativas y de sus implicaciones tanto económicas como sociales, lo cual redundará en unas mejores relaciones al amparo de la Teoría de los Stakeholders. Esto es, la comunicación social estratégica.

c) La idoneidad de la utilización de la memoria de sostenibilidad GRI (2007) por su referencia expresa al requisito de la inclusividad de los grupos de interés. Si relacionamos este aspecto con la condición de socio-propietario, y por tanto, parte de la cooperativa, observamos que desde la práctica se cumple este requisito fácilmente, más aún si reflexionamos sobre las diferentes funciones económicas donde también acaparan los socios otros papeles o condiciones diferentes: clientes, proveedores, empleados, entre otros.

Por todo lo comentado, consideramos justificada la selección de las memorias de sostenibilidad GRI para dar cuenta del desempeño social cooperativo y su utilidad como vía para mejorar la reputación de estas empresas. Si bien, nos disponemos en este último apartado a corroborar el papel que dentro del ámbito cooperativo desempeña la GRI dentro de las empresas cooperativas españolas. En este sentido, se ha procedido a revisar la base de datos ofrecida por la iniciativa GRI, filtrando esta información por el tipo de empresa y por el país de aplicación. Así, las cooperativas españolas que están haciendo uso de este estándar pueden ser observadas en la tabla $n^{0} 4^{5}$.

5.- La introducción del criterio delimitador relativo al tipo de empresa fue empleado por la GRI a partir del año 2012. A fecha de hoy, la única memoria de sostenibilidad que aparece en la base de datos del GRI para 2013 es la cooperativa Consum. 
Tabla 4. Cooperativas y GRI: una revisión

\begin{tabular}{|l|c|c|c|}
\hline Cooperativa & Título & Tipo & Nivel de aplicación 6 \\
\hline Consum & Sustainability Report 2011 & GRI - G3 & A+ \\
\hline La Fageda, SCCL & Memòria de Sostenibilitat 2011 & GRI - G3 & C \\
\hline $\begin{array}{l}\text { Linyola Agropecuària i Secció } \\
\text { de Crèdit SCLL }\end{array}$ & Memòria de Sostenibilitat 2012 & GRI - G3.1 & C \\
\hline Suara Cooperativa & Memòria de Sostenibilitat 2011 & GRI - Referenced & \\
\hline $\begin{array}{l}\text { Vinícola de Sarral i } \\
\text { Secció de Crèdit SCCL }\end{array}$ & Memòria de Gestió Sostenible Any 2011 & GRI - G3 & C \\
\hline
\end{tabular}

FUENTE: Elaborado a partir de GRI Database (2012).

El número de cooperativas que aparece en el anterior listado, 5, evidencia que resultan escasas las iniciativas de revelación social cooperativa, tal y como se ha venido reiterando a lo largo del trabajo, pero todavía menor en la utilización de la versión específica de la Guía GRI. Cuatro de las cinco cooperativas que han publicado su informe de sostenibilidad siguen el formato de la guía GRI G3/G3.1, si bien, existe un caso, en el que se usa un formato un poco diferente, se trata del denominado como GRI Referenced. En el análisis de tipos de informes de sostenibilidad y junto a los clásicos formatos G3/G3.1, se han incorporado aquellos otros informes que siguen las guías GRI pero sin índice de contenidos, los informes denominados como GRI Referenced. En cuanto al nivel de aplicación, igualmente observamos que sólo una cooperativa tiene el nivel máximo, $A$, siendo lo normal alcanzar el nivel básico catalogado como $\mathrm{C}$.

\section{5.- Conclusiones}

Una vez analizada la realidad multi-rol del socio cooperativo y la importancia de considerarlo como un stakeholder estratégico con el que es necesario mantener una comunicación transparente, bajo nuestro punto de vista, entendemos que la memoria de sostenibilidad de la GRI resulta fundamental para ayudar a la gestión cooperativa. La principal causa reside en la naturaleza compleja de los socios cooperativistas a los que se les atribuyen diversos roles que les sitúan en el centro de la gestión, como un agente de interés multifunción al que hay que atender desde diversas perspectivas. Su satisfacción por tanto se considera un objetivo estratégico. En este contexto, entendemos que la rendición de cuentas en el ámbito social debe tener al socio, que aúna y representa todas las posibles formas de

6.- El grado de aplicación depende del número de indicadores ofrecidos al elaborar la memoria, estableciéndose tres niveles, $C, B, A$, según mayor detalle de indicadores ofrecidos, el menor nivel de revelación sería el correspondiente a $C$ a diferencia del máximo que sería el nivel $A$. Igualmente, el distintivo "+" añadido al nivel de aplicación corresponde al hecho de que la memoria está verificada externamente. 


\section{LA REVELACIÓN SOCIAL A PARTIR DE LA GUÍA GRI DE LA ECONOMÍA SOCIAL: UNA HERRAMIENTA PARA MEJORAR LAS RELACIONES SOCIO-COOPERATIVA}

stakeholder, como principal interlocutor. Potenciar la comunicación como vía para reforzar las relaciones con cualquier colectivo es necesario, pero especialmente con los socios como grupo clave en estas empresas.

Por otro lado, la existencia de una iniciativa previa, como la GRI, específica para el ámbito de la Economía Social al que se adscriben las cooperativas, facilita su implementación y, por tanto, reduce los costes de posibles adaptaciones de cualquier marco global a este tipo de empresas. Las ventajas de su utilización son claras, solo falta una concienciación mayor en las cooperativas para que la herramienta sea utilizada y sepa comunicarse a los socios de forma adecuada porque los beneficios derivados de este esfuerzo parecen claros. Es decir, con este trabajo creemos justificado el necesario impulso a la promoción y a la difusión de la RSCoop entre las empresas cooperativas españolas, como vía para que la gestión cooperativa se impregne de una filosofía estratégica que permita aprovechar sus singularidades en el logro de un desempeño económico y social más adecuado.

Finalmente, debemos indicar que existe la necesidad de aprovechar la justificación teórica de nuestro estudio como argumento para tratar de difundir en el ámbito cooperativo las ventajas, en primer lugar, de su actuación social y, en segundo lugar, de la existencia de una guía que, de forma más específica, tiene en cuenta sus peculiaridades jurídicas. Probablemente un esfuerzo en la difusión de las ventajas de la divulgación con la herramienta GRI anime a las cooperativas españolas a sumarse a las pioneras y a competir en los mercados con la complicidad de los socios cooperativos como principal agente de interés.

\section{6.- Bibliografía}

AAIJAZ, N., SALLEH, S., IBRAHIM, M. \& DAHLAN, B. (2012): "Cooperative Social Responsibility: The Gap Model Approach and Empirical Derivation to Members' Satisfaction", 18 $8^{\text {th }}$ International Business Information Management Association Conference, Estambul, Turquía.

ARAGONÉS SIGNES, J. (1987): Cooperativismo, participación y poder, Centro de Educación Cooperativa, Valencia.

ARNOLD, D. (1993): The handbook of brand management, Pitman Publishing, London.

ATKINSON, A.A., WATERHOUSE, J.H. \& WELLS, R.B. (1997): "A Stakeholder Approach to Strategic Performance Measurement", Sloan Management Review, 38, 25-37.

BALMER, J.M.T. \& GREYSER, S.A. (2006): "Corporate marketing: integrating corporate identity, corporate branding, corporate communications, corporate image and corporate reputation", European Journal of Marketing, 40(7/8), 730-741. 
BELHAURI, A., BUENDÍA, I., LAPOINTE, M.J. \& TEMBLAY, B. (2012): "La responsabilidad social para las empresas: ¿un nuevo valor para las cooperativas", CIRIEC-España, Revista de Economía Pública, Social y Cooperativa, 53, 191-208.

BEST, R.J. (2004): Market-Based Management. Strategies for growing customer value and profitability, 3rd ed. International Edition, Prentice Hall, Upper Saddle River, NJ.

BIRTH, G., ILLIA, L., LURATI, F. \& ZAMPARINI, A. (2008): "Communicating CSR: practices among Switzerland's top 300 companies", Corporate Communications: An International Journal, 13 (2), 82-196.

BIJMAN, J. (2009): "Vertical coordination in the agrifood supply chain: structure and strategy of the agricultural cooperative". In: Advance in Supply Chain Analysis in Agro-Food Systems, Singular, Sao Paulo.

BROWN, L. \& HICKS, E. (2007): "Accounting for the Social: Incorporating Indicators of the Co-operative Difference into Strategic Planning", 1 st CIRIEC-International Research Conference on Social Economy, Victoria (Canada).

CABALLER MELLADO, V. (1992): Gestión y Contabilidad de Cooperativas Agrarias, Mundi-Prensa, Madrid.

CASTILLA POLO, F. \& GALLARDO VÁZQUEZ, D. (2003): "La comunicación voluntaria de información sobre intangibles: evidencias empíricas para el caso andaluz", Investigaciones europeas de dirección y economía de la empresa, 9 (2), 13-28.

CASTILLA POLO, F. \& GALLARDO VÁZQUEZ, D. (2011a): "La responsabilidad social en sociedades cooperativas. Especial referencia a la revelación social". En: VIII Reunión de Investigación en Contabilidad Social y Medioambiental - 8th Spanish Conference on Social and Environmental Accounting Research (Spanish CSEAR 2011).

CASTILLA POLO, F. \& GALLARDO VÁZQUEZ, D. (2011b): "Fondo y forma de la revelación social cooperativa", XVI Congreso de la Asociación Española de Contabilidad y Administración de Empresas, AECA, Granada.

CASTILLA POLO, F. \& GALLARDO VÁZQUEZ, D. (2014): "La revelación social en sociedades cooperativas. Evolución y situación actual", REVESCO, Revista de Estudios Cooperativos, 114, 7-34.

CECHIN, A., BIJMAN, J., PASCUCCI, S. \& OMTA, O. (2012): "Decomposing the member relationship in Agricultural Cooperatives: implications for commitment", Agribusiness, 29(1), 39-61.

COMISIÓN EUROPEA (2011): Estrategia renovada de la UE para 2011-2014 sobre la responsabilidad social de las empresas, Disponible en: http://eurlex.europa.eu/LexUriServ/LexUriServ.do?uri=COM:2011:0681:FIN:ES:PDF 
CONFEDERACIÓN EMPRESARIAL ESPAÑOLA DE LA ECONOMÍA SOCIAL (CEPES) (2007): Manual para el tutor del Programa RSE.COOP, Disponible en: http://www.cepes.es/istado.cfm?padre=389\&idSeccion=390\&idsec=sec_11

CHARTER, M. \& POLONSKY, M. (1999): Green Marketing. A global perspective on greening marketing practice, Green Leaf Publishing, Sheffield, UK.

CHAVES, R. \& SCHEDWY, R. (2004): "Gobierno, democracia y directivos en empresas cooperativas". CIRIEC-España, Revista de Economía Pública, Social y Cooperativa, 48, 5-10.

DE LOS RIOS BERJILLOS, A., RUIZ LOZANO, M., TIRADO VALENCIA, P. \& CARBONERO RUIZ, M. (2012): "Una aproximación a la relación entre información sobre responsabilidad social orientada al cliente y la reputación corporativa de las entidades financieras", Cuadernos de Economía y Dirección de Empresa, 15, 130-140.

DONALDSON, T. \& PRESTON, L. (1995): "The Stakeholders Theory of the corporation: concepts, evidence and implications", Academy of Management Review, 20, 65-91.

FENG, L. \& HENDRIKSE, G.W.J. (2012): "Chain interdependencies, measurement problems and efficient governance structure: cooperatives versus publicly listed firms", European Review of Agricultural Economics, 39, 241-255.

FREEMAN, R.E. (1984): Strategic management: a stakeholder approach, Pitman Publishing, Boston.

FROOMAN, J. (1999): "Stakeholder influence strategies", Academy of Management Journal, 42 (2), 191-206.

GALETZKA, M., GELDERS, K., VERCHENS, J.P. \& SEYDEL, E. (2008): "Transparency and performance communication: a case study of Dutch Railways", Corporate Communications: An International Journal, 13 (4), 433-447.

GALLARDO VÁZQUEZ, D., SÁNCHEZ HERNÁNDEZ, M.I. \& CASTILLA POLO, F. (2013): "Modelización estructural de la orientación a la responsabilidad social en las sociedades cooperativas y su impacto en los resultados", Información Comercial Española ICE, Revista de Economía, 873 (pendiente de publicación).

GARCÍA-GUTIÉRREZ FERNÁNDEZ, C. (1989): "El problema de la doble condición de los sociostrabajadores (socios-proveedores y socios-consumidores) ante la gerencia de la empresa cooperativa", REVESCO, Revista de Estudios Cooperativos, 56-57, 83-122.

GENOVART BALAGUER, J.I. (2012): Aspectos económicos-contables de las sociedades cooperativas, Tesis Doctoral, Universidad de las Islas Baleares.

GIJSELINCKX, C. (2009): "Co-operative stakeholders. Who counts in co-operatives, and how?", Working Papers on Social and Co-operative Entrepreneurship WP-SCE. 
GLOBAL REPORTING INITIATIVE (GRI) (2007): Ciclo Preparatorio para la Elaboración de Memorias de Sostenibilidad GRI. Edición Especial para la Economía Social, GRI, Colecciones de Aprendizaje. Disponible en: http://www.gri.org.

HANF, J. (2009): "Challenges of a vertical coordinated agri-food business for cooperatives", Journal of Cooperatives Studies, 42, 5-13.

HARRISON, J. \& FREEMAN, R.E. (1999): "Stakeholders, social responsability and performance: empirical evidence and theoretical perspectives", Academy of Management Journal, 42(5), 479-485.

HERNÁNDEZ ORTÍZ, M.J. (2001): Base social de las sociedades cooperativas almazareras. Un análisis cualitativo, Caja Rural de Jaén, Jaén.

HORRACH, P. \& SOCIAS, A. (2011): "La actitud de las empresas de economía solidaria frente a la divulgación de información sobre sostenibilidad desde el prisma de la Teoría de los Stakeholders o grupos de interés", Revista de Contabilidad, 14, 267-297.

HUETH, B. \& MARCOUL, P. (2009): "Incentive pay for CEOs in cooperatives firms", American Journal of Agricultural Economics, 91, 1218-1223.

JENSEN, M. (2010): "Value Maximization, Stakeholder Theory, and the Corporate Objective Function", Journal of Applied Corporate Finance, 2(1), 32-44.

JOHNSON, G. \& SCHOLES, K. (2001): Dirección estratégica, Prentice Hall, Madrid.

KMPG (2013): The KPMG Survey of Corporate Responsibility Reporting 2013. Disponible en: http://www.kpmg.com/BE/en/IssuesAndlnsights/ArticlesPublications/Documents/corporateresponsibility-reporting-survey-2013-exec-summary.pdf

KOTLER, P. (2003): Marketing Management, Prentice Hall, New Jersey.

LAPLUMNE, A.O., SONPAR, K. \& LITZ, R.A. (2008): "Stakeholder theory: Reviewing a theory that moves us", Journal of Management, 34(6), 1152-1189.

LIANG, Q. \& HENDRIKSE, G. (2013): "Cooperative CEO identity and efficient governance: member or outside CEO?", Agribusiness, 29, 23-38.

LICHTENSTEIN, D., DRUMWRIGHT, M. \& BRAIG, B. (2004): "The effect of corporate social responsibility on costumer donations to corporate-supported nonprofits", Journal of Marketing, 68, 16-32.

MAIGNAN, I. \& FERRELL, O.C. (2004): "Corporate social responsibility and marketing. An integrative framework", Journal of the Academic of Marketing Science, 32(1), 3-19.

MARÍN RIVES, L., ARCAS LARIO, N. \& CORTÉS GARCÍA, F.J. (2010): Guía práctica para la elaboración de memorias de RSE en cooperativas agroalimentarias, Fundación Cajamar, Colección Sostenibilidad, Almería.

MARK HERBERT, C. \& VON SCHANTZ, C. (2007): "Communicating Corporate Social Responsibility -Brand management", Electronic Journal of Business Ethics and Organization Studies, 12(2), 4-11. 
MONZÓN, J.L. \& ANTUÑANO, I. (2012): Cooperativismo y Responsabilidad Social de las Empresa. Veinte casos de cooperativas socialmente responsables, CIRIEC-España, Valencia.

MOOK, L., QUARTER, J. \& RICHMOND, B.J. (2007): What counts: Social accounting for nonprofits and cooperatives, Sigel Press.

MORSING, M. \& SCHULTZ, M. (2006): "Corporate social responsibility communication: Stakeholder information, response and involvement strategies", Business Ethics: An European Review, 15(4), 323-338.

MOZAS MORAL, A., POZA PÉREZ, J. \& VALLEJO MARTOS, M.C. (1997): "La gestión de recursos humanos en las sociedades cooperativas agrarias: un estudio empírico", REVESCO, Revista de Estudios Cooperativos, 63, 93-119.

MOZAS MORAL, A., PUENTES POYATOS, R. \& BERNAL JURADO, E. (2010): "La E-Corporate Social Responsibility en las sociedades cooperativas agrarias andaluzas", CIRIEC-España, Revista de Economía Pública, Social y Cooperativa, 67, 187-212.

MOYANO FUENTES, J., PUIG BLANCO, F. \& BRUQUE CÁMARA, S. (2008): "Los determinantes de la competitividad en cooperativas", CIRIEC-España, Revista de Economía Social, Pública y Cooperativa, 61, 233-249.

MUGARRA ELORRIAGA, A. (2001): "Responsabilidad y Balance Social hoy en día: Un reto para las Cooperativas", CIRIEC-España, Revista de Economía Pública, Social y Cooperativa, 39, 25-50.

NILSSON, J., SVENDSEN, G.L.H. \& SVENDSEN, G.T. (2012): "Are large and complex agricultural cooperatives losing their social capital?", Agribusiness, 28(2),187-204.

ORTS, E.W. \& STRUDLER, A. (2009): "The ethical and environmental limits of stakeholder theory", Business Ethics Quarterly, 12(2), 215-233.

PEDREGOSA ORGEGA, C. (2009): "Modelos teóricos que nos ayudan a comprender el gobierno de las sociedades cooperativas, una apuesta por el enfoque de los stakeholders", Gestión Joven, 4, 5.

PEDREGOSA ORTEGA, C. \& GARCÍA MARTÍ, E. (2011): "El gobierno en la sociedad cooperativa como base de cohesión social: el caso de una sociedad cooperativa agraria", REVESCO, Revista de Estudios Cooperativos, 106, 7-32.

PÉREZ FERNÁNDEZ, E. (2002): "La participación como elemento constitutivo de las empresas de la Nueva Economía Social", CIRIEC-España, Revista de economía pública, social y cooperativa, 40, 141-163.

PUENTES POYATOS, R., VELASCO GÁMEZ, M.M. \& HERNÁNDEZ VILAR, J. (2009): "El buen gobierno corporativo en las sociedades cooperativas", REVESCO, Revista de Estudios Cooperativos, 98, 118-140.

RAMONET, I. (2002): Guerras del siglo XXI. Nuevos miedos nuevas amenazas, Mondadori, Barcelona. 
REGUANT, D.F. (2012): "Las cooperativas en un entorno competitivo", Agricolae, 1, 105-110.

REINHARD, K., OSBURG, T. \& TOWSEND, R. (2010): "Introducing corporate social responsibility as component in cooperative education: Results from a student research project in Germany and the United States supported by Intel Corporation", Asia - Pacific Journal of Cooperative Education, $11(1), 1-12$.

RETOLAZA, J.L. \& SAN-JOSE, L. (2011): "Social economy and stakeholder theory, an integrative framework for socialization of the capitalism", CIRIEC-España, Revista de Economía Pública, Social y Cooperativa, 73, 193-212.

RONDOT, S. \& BOUCHARD, M. (2003): "L'evaluation en économie sociale. Petit aide-mémorie". Alliance de reserche universités-comunatutés en économie sociale, Cahiers de recherche, ${ }^{\circ}$ R032003, mars, 35 .

RUÍZ JIMÉNEZ, C. \& GARCÍA MARTÍ, E. (2002); "La ética de la sociedad cooperativa agraria en la relación con sus grupos de interés", REVESCO, Revista de Estudios Cooperativos, 76, 89-108.

SERVER, R.J. \& CAPO, J. (2011): "The interrelationship between the demands of Corporate Social Responsibility and co-operative principles and values", CIRIEC-España, Revista de Economía Pública, Social y Cooperativa, 73, 213-232.

SPEAR, R. (2004): "Governance in Democratic Member Based Organizations", Annals of Public and Cooperative Economics, 75(1), 33-60.

TRANSPARENCIA INTERNACIONAL (2013): Principios de transparencia y prevención de la corrupción para las empresas. Disponible en http://www.transparencia.org.es.

UNIÓN EUROPEA (UE) (2013): Informe sobre la responsabilidad social de las empresas: comportamiento responsable y transparente de las empresas y crecimiento sostenible. Ponente: Baldassarre, R. Documento A7-0017/2013.

VARGAS SÁNCHEZ, A. (2004): "Empresas cooperativas, ventaja competitiva y tecnologías de la información", CIRIEC-España, Revista de Economía Pública, Social y Cooperativa, 49, 13-29.

VARGAS SÁNCHEZ, A. \& VACAS ACOSTA, R.M. (2005): "Responsabilidad Social Corporativa y cooperativismo: Vínculos y potencialidades", CIRIEC-España, Revista de Economía Pública, Social y Cooperativa, 53, 241-260.

WERTHER, W.B. \& CHANDLER, D. (2011): Strategic Corporate Social Responsibility, Sage Publications Inc., California.

ZHAO, L. \& GIJSELINCKX, C. (2011): "Multistakeholder co-operatives in China: a resource mix structure approach", Social Enterprise Journal, 7(3), 259-279. 